\title{
Universal finite-size scaling analysis of Ising models with long-range interactions at the upper critical dimensionality: Isotropic case
}

\author{
Daniel Grüneberg* and Alfred Huchti \\ Fakultät für Naturwissenschaften, Theoretische Physik, \\ Universität Duisburg-Essen, D-47048 Duisburg, Germany
}

\begin{abstract}
We investigate a two-dimensional Ising model with long-range interactions that emerge from a generalization of the magnetic dipolar interaction in spin systems with in-plane spin orientation. This interaction is, in general, anisotropic whereby in the present work we focus on the isotropic case for which the model is found to be at its upper critical dimensionality. To investigate the critical behavior the temperature and field dependence of several quantities are studied by means of Monte Carlo simulations. On the basis of the Privman-Fisher hypothesis and results of the renormalization group the numerical data are analyzed in the framework of a finite-size scaling analysis and compared to finite-size scaling functions derived from a Ginzburg-Landau-Wilson model in zero mode (meanfield) approximation. The obtained excellent agreement suggests that at least in the present case the concept of universal finite-size scaling functions can be extended to the upper critical dimensionality.
\end{abstract}

PACS numbers: 05.50.+q, 05.70.Fh, 75.10.Hk, 89.75.Da

\section{INTRODUCTION}

In the last decade, spin models with long-range interactions were the subject of several extensive Monte Carlo studies. Utilizing an efficient cluster algorithm [1] these studies were addressed to the verification of some unproved predictions on the critical behavior of spin models with algebraically decaying long-range interactions 2]. Furthermore, the crossover from Ising-like to classical critical behavior was investigated 3, 4] and first numerical results on the critical behavior of the dipolar in-plane Ising (DIPI) model were obtained [5]. This twodimensional model displays a strongly anisotropic phase transition, i.e. , the correlation lengths in direction parallel and perpendicular to spin orientation diverge in the infinite system (let $t>0$ ) as 5

$$
\xi_{\|}^{(\infty)}(t) \sim \hat{\xi}_{\|} t^{-\nu_{\|}}, \quad \xi_{\perp}^{(\infty)}(t) \sim \hat{\xi}_{\perp} t^{-\nu_{\perp}}
$$

at the critical point, where both $\hat{\xi}_{\|} \neq \hat{\xi}_{\perp}$ and $\nu_{\|} \neq \nu_{\perp}$, and $t \equiv\left(T-T_{\mathrm{c}}\right) / T_{\mathrm{c}}$ denotes the reduced temperature. Except the anisotropy exponent $\theta=\nu_{\|} / \nu_{\perp}$ neither any numerical estimates of the critical exponents exist for the DIPI model, nor is it clear whether the model exhibits Lifshitz type critical behavior [6] as it is observed, e.g. , in the anisotropic next nearest neighbor Ising (ANNNI) model [7, 8, 9].

To address such questions in a broader context we present a two-dimensional long-range spin model that arises from generalizing the DIPI model by introducing an additional parameter. Assuming $L_{\|} \times L_{\perp}$ geometry and periodic boundary conditions this model is described

*Electronic address: daniel@thp.Uni-Duisburg.de

$\dagger$ Electronic address: fred@thp.Uni-Duisburg.de

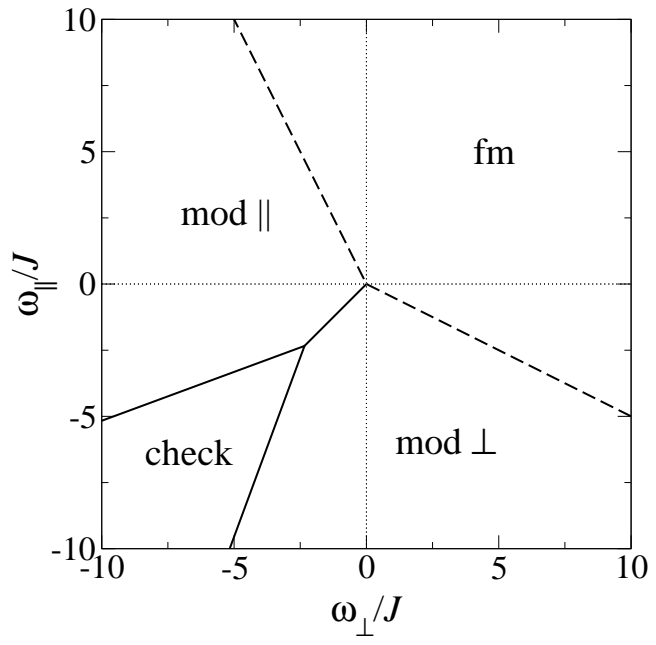

Figure 1: The model (Eqs. (2)) exhibits a ferromagnetic ground state in the region denoted by "fm". The dashed lines $\omega_{\|}=-2 \omega_{\perp}>0$ and $-2 \omega_{\|}=\omega_{\perp}>0$, respectively, and the origin $\omega_{\|}=\omega_{\perp}=0$ also represent ferromagnetic ground states. All other regions correspond to modulated spin configurations (see text).

by the Hamiltonian

$$
\mathcal{H}=-\frac{1}{2} \sum_{i \neq j} s_{i} J\left(\mathbf{r}_{i j}\right) s_{j}-B \sum_{i} s_{i}
$$

with magnetic Ising spin variables $s_{i}= \pm 1$, the spin-spin distance vector $\mathbf{r}_{i j}$, and an external field $B$. The pair coupling $J(\mathbf{r})$ is given by

$$
J(\mathbf{r})=J \delta_{|\mathbf{r}|, 1}+\frac{\omega_{\|} r_{\|}^{2}+\omega_{\perp} r_{\perp}^{2}}{|\mathbf{r}|^{5}}
$$

and consists of both a ferromagnetic short-range nearest neighbor exchange coupling with the coupling constant 
$J \geq 0$ and a long-range contribution, where $r_{\|}$and $r_{\perp}$ are the components of the vector $\mathbf{r}$ parallel and perpendicular to spin orientation. Using this general form of the pair coupling $J(\mathbf{r})$, several well known spin systems can be mapped onto this model by making an explicit choice of the parameters $\omega_{\|}, \omega_{\perp}$, and $J$. With $\omega_{\|}=-2 \omega_{\perp}>0$, and for symmetry reasons $-2 \omega_{\|}=\omega_{\perp}>0$, and $J>0$ the DIPI model is recovered, and if $\omega_{\|}=\omega_{\perp}>0$ and $J=0$ Eq. (2a) corresponds to an Ising model with an isotropic ferromagnetic long-range interaction algebraically decaying as $J(\mathbf{r}) \propto|\mathbf{r}|^{-3}$. Another special case is the dipolar Ising model with perpendicular spin orientation [10, 11] that can be obtained for $\omega_{\|}=\omega_{\perp}<0$ and $J>0$.

Figure 1 shows the ground state phase diagram of the model whereby we took into account four different ground state spin configurations: the ferromagnetic state (fm) where all spins point to the same direction, the totally antiferromagnetic state that is referred to as checkerboard state (check), and commensurate stripe domain states with a domain wall orientation parallel $(\bmod \|)$ and perpendicular $(\bmod \perp)$ to spin orientation and the periods $N_{\|, \perp}$. Dependent on the values of the quotients $\omega_{\|} / J$ and $\omega_{\perp} / J$ all considered spin configurations were found as stable ground states. Due to symmetry reasons the arrangement of the corresponding phases in Fig. 1 is symmetric with respect to the line $\omega_{\|}=\omega_{\perp}$. The periods $N_{\|, \perp}$ of the stripe domain states diverge when approaching the dashed lines in Fig. 10

The region in parameter space where the model displays a ferromagnetic ground state is of particular interest to us. In that region the observed phase transitions are isotropic (anisotropic) when $\omega_{\|}=\omega_{\perp}\left(\omega_{\|} \neq \omega_{\perp}\right)$ whereby in the present work we draw our attention to the isotropic ferromagnetic long-range case

$$
\omega_{\|}=\omega_{\perp}>0, \quad J=0
$$

before we turn to the case of an anisotropic pair coupling 12]. It is known that the upper critical dimension of long-range spin models with ferromagnetic interactions decaying as $J(\mathbf{r}) \propto|\mathbf{r}|^{-(d+\sigma)}$ is given by $d_{\mathrm{u}}=2 \sigma$ 13]. Comparison with the pair coupling $J(\mathbf{r})$ defined in Eq. (2b) yields in the isotropic case, Eq. (3), $\sigma=1$ for a two-dimensional system and consequently $d=d_{\mathrm{u}}=2$.

So in this paper we investigate the critical behavior of the model at its borderline dimensionality $d_{\mathrm{u}}$ by means of Monte Carlo (MC) simulations and finite-size scaling methods. For that purpose in the following Section III the finite-size scaling form of the free energy density is discussed and the finite-size scaling relations of the considered quantities are derived as they are used for the finite-size scaling analysis. These relations define finitesize scaling functions for which in Section III we evaluate analytical expressions in the framework of the so-called zero mode theory that is based on the Ginzburg-LandauWilson (GLW) model. In the last Section IV the zero mode results are compared to numerical data within a finite-size scaling analysis.

\section{FINITE-SIZE SCALING RELATIONS}

To study the critical properties of the model in the isotropic long-range case we have carried out a finitesize scaling analysis of MC data. This analysis requires the finite-size scaling relations of the quantities that were considered in the simulations.

Via a renormalization group approach, Luijten and Blöte 2] derived the scaling form of the free energy density of $O(n)$ spin models with ferromagnetic long-range interactions decaying as $J(\mathbf{r}) \propto|\mathbf{r}|^{-(d+\sigma)}$. At the upper critical dimension, that is given by $d_{\mathrm{u}}=2$ for $\sigma=1$ (see Sec. I), the singular part of the reduced free energy density was found to scale as $(n=1)$

$$
\begin{aligned}
f_{s}(t, h ; L) \sim & L^{-2} \widetilde{f}\left(u^{-\frac{1}{3}} L \ln ^{\frac{1}{6}}\left(\frac{L}{L_{0}^{\prime}}\right)\left[t-v^{\prime} L^{-1} \ln ^{-\frac{2}{3}}\left(\frac{L}{L_{0}^{\prime}}\right)\right],\right. \\
& \left.L^{\frac{3}{2}} \ln ^{\frac{1}{4}}\left(\frac{L}{L_{0}^{\prime}}\right) h\right)
\end{aligned}
$$

with the reduced temperature $t$, the reduced external field $h \equiv \beta B$ where $\beta \equiv 1 /\left(k_{\mathrm{B}} T\right)$ denotes the inverse temperature (we set $k_{\mathrm{B}}=1$ throughout this paper), and the so-called dangerous irrelevant variable $u$ [14, 15]. Note that we rewrote the formula given in Ref. [2] in terms of the parameters $v^{\prime}$ and $L_{0}^{\prime}$, where $L_{0}^{\prime}$ can be regarded as a reference length that fixes the length scale in the logarithms (see also Ref. [16]), and we absorbed constant factors into $\tilde{f}$. The symbol $\sim$ means "asymptotically equal" and, unless stated otherwise, refers to the limit $(t, h, L) \rightarrow(0,0, \infty)$ with $t L \ln ^{\frac{1}{6}}(L)$ and $h L^{\frac{3}{2}} \ln ^{\frac{1}{4}}(L)$ fixed (cf. Eq. (7)).

Proceeding from Eq. (4) we adapt the Privman-Fisher hypothesis [17] and propose the finite-size scaling form of the singular part of the reduced free energy density

$$
f_{s}(t, h ; L) \sim L^{-2} Y\left(x_{\mathrm{rg}}, y_{\mathrm{rg}}\right)
$$

with the universal finite-size scaling (UFSS) function $Y(x, y)$. The arguments of this function correspond to the temperature scaling variable

$$
x_{\mathrm{rg}}=C_{1} \hat{t} L \ln ^{\frac{1}{6}}\left(\frac{L}{L_{0}}\right)
$$

with the shifted reduced temperature

$$
\hat{t}=t-v L^{-1} \ln ^{-\frac{2}{3}}\left(\frac{L}{L_{0}}\right),
$$

and the field scaling variable

$$
y_{\mathrm{rg}}=C_{2} h L^{\frac{3}{2}} \ln ^{\frac{1}{4}}\left(\frac{L}{L_{0}}\right),
$$

whereby $C_{1}$ and $C_{2}$ are nonuniversal metric factors. Let us note that we have replaced the constants $v^{\prime}$ and $L_{0}^{\prime}$ which arise from the renormalization group by the constants $v$ and $L_{0}$ that will be used as fit parameters in the finite-size scaling analysis.

It is also important to point out that the terms that result from $v$ and $L_{0}$ in the temperature scaling variable 
$x_{\mathrm{rg}}$ and the field scaling variable $y_{\mathrm{rg}}$ are merely corrections since they do not contribute to the leading orders in the expansions

$$
\begin{aligned}
\hat{t} L \ln ^{\frac{1}{6}}\left(\frac{L}{L_{0}}\right) \stackrel{L \rightarrow \infty}{=} & t L \ln ^{\frac{1}{6}}(L)+v \mathcal{O}\left(\ln ^{-\frac{1}{2}}(L)\right) \\
& +t L \ln \left(L_{0}\right) \mathcal{O}\left(\ln ^{-\frac{5}{6}}(L)\right) \\
h L^{\frac{3}{2}} \ln ^{\frac{1}{4}}\left(\frac{L}{L_{0}}\right) \stackrel{L \rightarrow \infty}{=} & h L^{\frac{3}{2}} \ln ^{\frac{1}{4}}(L) \\
& +h L^{\frac{3}{2}} \ln \left(L_{0}\right) \mathcal{O}\left(\ln ^{-\frac{3}{4}}(L)\right) .
\end{aligned}
$$

However, due to the slow convergence of the logarithms appearing at the upper critical dimension these corrections are substantial for the quality of the data collapse in the finite-size scaling analysis, as will be discussed in Sec. IV (see also Sec. IV(B) in [2], and [16]).

In the following we derive the finite-size scaling forms of the quantities that were considered in the simulations. Let

$$
\bar{s}=\frac{1}{L^{d}} \sum_{j=1}^{L^{d}} s_{j}
$$

denote the average of the spin variables $s_{j}$, these quantities are the magnetization $m(t, h ; L)=\langle\bar{s}\rangle$ and the susceptibility $\chi(t, h ; L)=\beta L^{d}\left(\left\langle\bar{s}^{2}\right\rangle-\langle\bar{s}\rangle^{2}\right)$, whose finite-size scaling forms can be obtained by taking derivatives of the singular part of the reduced free energy density, Eq. (5), according to

$$
\begin{aligned}
m(t, h ; L) & =-\frac{\partial}{\partial h} f_{s}(t, h ; L) \\
& \sim C_{2} L^{-\frac{1}{2}} \ln ^{\frac{1}{4}}\left(\frac{L}{L_{0}}\right) Y_{m}\left(x_{\mathrm{rg}}, y_{\mathrm{rg}}\right), \\
\beta^{-1} \chi(t, h ; L) & =-\frac{\partial^{2}}{\partial h^{2}} f_{s}(t, h ; L) \\
& \sim C_{2}^{2} L \ln ^{\frac{1}{2}}\left(\frac{L}{L_{0}}\right) Y_{\chi}\left(x_{\mathrm{rg}}, y_{\mathrm{rg}}\right) .
\end{aligned}
$$

We also consider the dimensionless Binder cumulant 18] $U(t ; L)=1-\left\langle\bar{s}^{4}\right\rangle /\left(3\left\langle\bar{s}^{2}\right\rangle^{2}\right)$ that can be evaluated from the susceptibility $\chi(t, h ; L)$ and the nonlinear susceptibility $\chi^{(\mathrm{nl})}(t, h ; L)$ using the identity [19]

$$
U(t ; L)=-\frac{\chi^{(\mathrm{nl})}(t, 0 ; L)}{3 \beta L^{2} \chi^{2}(t, 0 ; L)},
$$

where $\chi^{(\mathrm{nl})}(t, h ; L)$ is given by

$$
\begin{aligned}
\beta^{-3} \chi^{(\mathrm{nl})}(t, h ; L) & =-\frac{\partial^{4}}{\partial h^{4}} f_{s}(t, h ; L) \\
& \sim C_{2}^{4} L^{4} \ln \left(\frac{L}{L_{0}}\right) Y_{\chi^{(\mathrm{nl})}}\left(x_{\mathrm{rg}}, y_{\mathrm{rg}}\right) .
\end{aligned}
$$

Hence, the Binder cumulant scales as

$$
U(t ; L) \sim Y_{U}\left(x_{\mathrm{rg}}\right),
$$

where $Y_{U}(x) \equiv-Y_{\chi^{(\mathrm{nl})}}(x, 0) /\left(3 Y_{\chi}^{2}(x, 0)\right)$. Since the ensemble averages $\left\langle\bar{s}^{2 m+1}\right\rangle$ with $m \in \mathbb{N}_{0}$ vanish in the absence of an external field, we, in addition, analyze the magnetization $m_{\mathrm{abs}}(t, h ; L)=\langle|\bar{s}|\rangle$ and the susceptibility $\chi_{\text {abs }}(t, h ; L)=\beta L^{d}\left(\left\langle\bar{s}^{2}\right\rangle-\langle|\bar{s}|\rangle^{2}\right)$. It is understood that these quantities also fulfill the finite-size scaling forms of $m(t, h ; L)$ and $\chi(t, h ; L)$, Eqs. (9), respectively with the corresponding UFSS functions $Y_{m_{\text {abs }}}(x, y)$ and $Y_{\text {Хabs }}(x, y)$.

\section{MEAN-FIELD THEORY}

An appropriate description of spin systems of dimensions $L \times L \times \cdots \times L=L^{d}$ with mean-field-like (classical) critical behavior can be achieved by the mean-field theory employed by Brézin and Zinn-Justin [20] and Rudnick et al. [21]. This theory, also known as zero mode approximation, yields in contrast to conventional meanfield theories a rounded transition for finite systems. In the thermodynamic limit $L \rightarrow \infty$ the usual power laws with the expected mean-field values of the critical exponents can be recovered.

In the following, this theory is reviewed and used to evaluate analytical expressions for the finite-size scaling functions defined in the preceding section in order to compare them to the numerical data, as it is demonstrated in Sec. IV The basis of this evaluation is the reduced GLW Hamiltonian in momentum space that corresponds to the underlying spin system with a long-range interaction $J(\mathbf{r}) \propto|\mathbf{r}|^{-(d+\sigma)}$. It is given by (see, e. g. , Ref. 22])

$$
\begin{aligned}
\overline{\mathcal{H}}= & L^{d}\left(\frac{1}{2} \sum_{\mathbf{k}}\left(r+A_{\sigma}|\mathbf{k}|^{\sigma}\right) \varphi_{\mathbf{k}} \varphi_{-\mathbf{k}}-h \varphi_{\mathbf{0}}\right. \\
& \left.+\frac{u}{4 !} \sum_{\mathbf{k}_{1}} \sum_{\mathbf{k}_{2}} \sum_{\mathbf{k}_{3}} \varphi_{\mathbf{k}_{1}} \varphi_{\mathbf{k}_{2}} \varphi_{\mathbf{k}_{3}} \varphi_{-\mathbf{k}_{1}-\mathbf{k}_{2}-\mathbf{k}_{3}}\right)
\end{aligned}
$$

with the temperature-like parameter $r \propto T-T_{\mathrm{c}}^{\mathrm{mf}}$ that measures the deviation of the temperature from the mean-field critical temperature $T_{\mathrm{c}}^{\mathrm{mf}}$, the reduced external field $h$, and the dangerous irrelevant variable $u>0$. Each sum in Eq. (13) runs for each component $k_{j}$ of $\mathbf{k}$ over integer multiples of $2 \pi / L$ up to a momentum space cutoff $k_{\Lambda}=\pi / a\left(\left|k_{j}\right| \leq k_{\Lambda}\right)$ with the lattice constant $a$.

The essential step of the zero mode approximation is the neglection of all modes except the zero mode $\varphi \equiv$ $\varphi_{\mathbf{0}}$ in Eq. (13). This leads to the reduced zero mode Hamiltonian (see, e.g. , Ref. 21])

$$
\overline{\mathcal{H}}_{0}(\varphi)=L^{d}\left(\frac{r}{2} \varphi^{2}+\frac{u}{4 !} \varphi^{4}-h \varphi\right) .
$$

with the corresponding partition function

$$
\mathcal{Z}_{0}=\int_{-\infty}^{\infty} \mathrm{d} \varphi e^{-\overline{\mathcal{H}}_{0}(\varphi)} .
$$

So the normalized probability distribution of the zero mode is given by

$$
\mathcal{P}_{0}(\varphi)=\frac{1}{\mathcal{Z}_{0}} e^{-\overline{\mathcal{H}}_{0}(\varphi)}
$$


and it can be used to evaluate averages of the form

$$
\langle g(\varphi)\rangle_{0}=\int_{-\infty}^{\infty} \mathrm{d} \varphi g(\varphi) \mathcal{P}_{0}(\varphi) .
$$

A further central quantity is the reduced free energy density that is given by $f_{0}=-L^{-d} \ln \left(\mathcal{Z}_{0}\right)$ within the zero mode approximation [19, 20]. Using this expression and the zero mode partition function defined in Eq. (15), the rescaling (see, e.g. , Ref. [20])

$$
\varphi \rightarrow\left(u L^{d}\right)^{-\frac{1}{4}} \varphi
$$

immediately yields the zero mode finite-size scaling form of $f_{0}$. It reads

$$
f_{0}(r, h ; L)=L^{-d} \tilde{f}_{0}\left(x_{\mathrm{mf}}, y_{\mathrm{mf}}\right)+c(L)
$$

with the mean-field temperature and field scaling variables

$$
x_{\mathrm{mf}}=r u^{-\frac{1}{2}} L^{\frac{d}{2}}, \quad y_{\mathrm{mf}}=h u^{-\frac{1}{4}} L^{\frac{3 d}{4}},
$$

and an additive term $c(L)$ that is without significance in the following since it is absent after taking derivatives of $f_{0}(r, h ; L)$ with respect to $r$ or $h$. Instead we focus on the finite-size scaling function

$$
\tilde{f}_{0}(x, y)=-\ln \left(\int_{-\infty}^{\infty} \mathrm{d} \varphi e^{-\left(\frac{x}{2} \varphi^{2}+\frac{1}{24} \varphi^{4}-y \varphi\right)}\right)
$$

from which, as seen in the preceding section, the finitesize scaling functions of other quantities like the magnetization $m$ and the susceptibility $\chi$ follow.

The asymptotics of this function are given by

$$
\begin{aligned}
& \tilde{f}_{0}(x, 0) \stackrel{x \rightarrow-\infty}{\sim}-\frac{3}{2} x^{2}+\frac{1}{2} \ln \left(\frac{|x|}{4 \pi}\right) \\
& \tilde{f}_{0}(x, 0) \stackrel{x \rightarrow+\infty}{\sim} \frac{1}{2} \ln \left(\frac{x}{2 \pi}\right) \\
& \tilde{f}_{0}(0, y) \stackrel{y \rightarrow \pm \infty}{\sim}-\left(\frac{81}{32}\right)^{\frac{1}{3}}|y|^{\frac{4}{3}} .
\end{aligned}
$$

Following the convention suggested in Ref. 23], the normalized finite-size scaling function $Y^{\mathrm{mf}}(x, y)$ of the reduced free energy density should be defined such that their asymptotics read

$$
\begin{aligned}
& Y^{\mathrm{mf}}(x, 0) \stackrel{x \rightarrow-\infty}{\sim}-x^{2} \\
& Y^{\mathrm{mf}}(0, y) \stackrel{y \rightarrow \pm \infty}{\sim}-|y|^{\frac{4}{3}}
\end{aligned}
$$

instead of the leading orders in Eqs. (22). This requirement fixes some arbitrariness of the reduced free energy density finite-size scaling function and can be fulfilled by a rescaling of the parameters $r$ and $u$, which are the only phenomenological quantities entering the reduced zero mode Hamiltonian, Eq. (14). Replacing $x$ and $y$ in Eqs. (22) explicitly with the mean-field scaling variables $x_{\mathrm{mf}}$ and $y_{\mathrm{mf}}$ from Eqs. (20), the rescaling

$$
r \rightarrow \frac{3 \sqrt{3}}{4} r, \quad u \rightarrow \frac{81}{32} u
$$

leads to a cancellation of the corresponding coefficients of $-x_{\mathrm{mf}}^{2}$ and $-\left|y_{\mathrm{mf}}\right|^{\frac{4}{3}}$ and one obtains the desired asymptotics. It is important to note that we do not alter the definitions of $x_{\mathrm{mf}}$ and $y_{\mathrm{mf}}$ due to this rescaling, but the scaling function itself. Starting from the zero mode partition function, Eq. (15), with the rescaled parameters $r$ and $u$, we, after the procedure discussed above, finally end up with

$$
Y^{\mathrm{mf}}(x, y)=-\ln \left(\Xi_{0}(x, y)+\Xi_{0}(x,-y)\right)
$$

where

$$
\Xi_{m}(x, y)=\int_{0}^{\infty} \mathrm{d} \varphi \varphi^{m} e^{-\left(\frac{3 \sqrt{3}}{8} x \varphi^{2}+\frac{27}{256} \varphi^{4}-y \varphi\right)},
$$

instead of $\tilde{f}_{0}(x, y)$ given in Eq. (21).

Having defined the reduced free energy density finitesize scaling function, in the following the finite-size scaling forms and the corresponding zero mode finite-size scaling functions of the quantities considered in the preceding section will be derived. Since the quantities $m_{\text {abs }}(t, h ; L)$ and $\chi_{\text {abs }}(t, h ; L)$ cannot be evaluated by taking derivatives of the reduced free energy density, we instead make use of the average defined in Eq. (17). Setting here $g(\varphi)=\varphi^{m}$ and $g(\varphi)=|\varphi|^{m}$, respectively, the rescaling of the parameters $r$ and $u$ [Eq. (24)] and the rescaling of the zero mode (Eq. (18) ) immediately yields the finite-size scaling forms of these averages. They read

$$
\begin{aligned}
\left\langle\varphi^{m}\right\rangle_{0} & =\left(u L^{d}\right)^{-\frac{m}{4}} Y^{(m), \mathrm{mf}}\left(x_{\mathrm{mf}}, y_{\mathrm{mf}}\right), \\
\left\langle|\varphi|^{m}\right\rangle_{0} & =\left(u L^{d}\right)^{-\frac{m}{4}} Y_{\mathrm{abs}}^{(m), \mathrm{mf}}\left(x_{\mathrm{mf}}, y_{\mathrm{mf}}\right)
\end{aligned}
$$

with the finite-size scaling functions

$$
\begin{aligned}
Y^{(m), \mathrm{mf}}(x, y) & =\frac{\Xi_{m}(x, y)+(-1)^{m} \Xi_{m}(x,-y)}{\Xi_{0}(x, y)+\Xi_{0}(x,-y)},(28 \mathrm{a}) \\
Y_{\mathrm{abs}}^{(m), \mathrm{mf}}(x, y) & =\frac{\Xi_{m}(x, y)+\Xi_{m}(x,-y)}{\Xi_{0}(x, y)+\Xi_{0}(x,-y)} .
\end{aligned}
$$

Since, as discussed in Ref. 20], the zero mode $\varphi$ is related to the order parameter field $\phi(\mathbf{r})$ in real space via

$$
\varphi=\frac{1}{L^{d}} \sum_{j=1}^{L^{d}} \phi\left(\mathbf{r}_{j}\right)
$$

and consequently corresponds to the average order parameter per volume, one immediately obtains the finitesize scaling forms of the quantities defined in Sec. II] using Eqs. 27]. Due to this correspondence, the magnetization $m(r, h ; L)$ and the susceptibility $\chi(r, h ; L)$ are given by

$$
\begin{aligned}
m(r, h ; L) & =\langle\varphi\rangle_{0} \\
\beta^{-1} \chi(r, h ; L) & =L^{d}\left(\left\langle\varphi^{2}\right\rangle_{0}-\langle\varphi\rangle_{0}^{2}\right)
\end{aligned}
$$

and therefore, according to Eq. (27a), scale as 


$$
\begin{aligned}
m(r, h ; L) & =u^{-\frac{1}{4}} L^{-\frac{d}{4}} Y_{m}^{\mathrm{mf}}\left(x_{\mathrm{mf}}, y_{\mathrm{mf}}\right) \\
\beta^{-1} \chi(r, h ; L) & =u^{-\frac{1}{2}} L^{\frac{d}{2}} Y_{\chi}^{\mathrm{mf}}\left(x_{\mathrm{mf}}, y_{\mathrm{mf}}\right)
\end{aligned}
$$

with the zero mode finite-size scaling functions $Y_{m}^{\mathrm{mf}}(x, y)$ and $Y_{\chi}^{\mathrm{mf}}(x, y)$. The finite-size scaling forms of the quantities

$$
\begin{aligned}
m_{\mathrm{abs}}(r, h ; L) & =\langle|\varphi|\rangle_{0} \\
\beta^{-1} \chi_{\mathrm{abs}}(r, h ; L) & =L^{d}\left(\left\langle\varphi^{2}\right\rangle_{0}-\langle|\varphi|\rangle_{0}^{2}\right)
\end{aligned}
$$

are identical to Eqs. (31), respectively, with the corresponding finite-size scaling functions $Y_{m_{\mathrm{abs}}}^{\mathrm{mf}}(x, y)$ and $Y_{\chi \mathrm{abs}}^{\mathrm{mf}}(x, y)$. A further quantity of interest is the dimensionless Binder cumulant

$$
U(r ; L)=1-\left.\frac{\left\langle\varphi^{4}\right\rangle_{0}}{3\left\langle\varphi^{2}\right\rangle_{0}^{2}}\right|_{h=0}
$$

for which within the zero mode approximation one obtains the scaling form

$$
U(r ; L)=Y_{U}^{\mathrm{mf}}\left(x_{\mathrm{mf}}\right)
$$

with the finite-size scaling function $Y_{U}^{\mathrm{mf}}(x)$.

All zero mode finite-size scaling functions $Y_{i}^{\mathrm{mf}}(x, y)$ can be expressed as combinations of the functions $\Xi_{m}(x, y)$ defined in Eq. (26) with $m=\{0,1,2,4\}$. Since to analyze the critical behavior, as it is done in the MC simulations, either the temperature scaling variable or the field scaling variable is kept at its critical point value, it is sufficient to evaluate $\Xi_{m}(x, 0)$ and $\Xi_{m}(0, y)$ for the pertinent values of $m$. Analytical expressions for the needed functions can be found in the Appendix.

\section{MONTE CARLO RESULTS}

In this section we present the results of MC simulations that were carried out for the model [Eq. (2)] with $\left(\omega_{\|}, \omega_{\perp}, J\right)=(1,1,0)$. We have used the Wolff cluster algorithm 24] for long-range spin models proposed by Luijten and Blöte [1]. To study the properties of the model in the presence of an external magnetic field, a histogram reweighting technique [25] was used.

In the simulations quadratic spin systems with $L:=$ $L_{\|}=L_{\perp}=\{32,64,128,256,512,1024\}$ were considered and we started with recording the magnetization $m_{\text {abs }}$, the susceptibilities $\chi$ and $\chi_{\text {abs }}$, and the Binder cumulant $U$ at zero field for various temperatures close to the corresponding mean-field critical temperature $T_{\mathrm{c}}^{\mathrm{mf}}$. This temperature is given by $T_{\mathrm{c}}^{\mathrm{mf}}=\widetilde{J}(\mathbf{0})$ (see, e.g. , Ref. [26]) where $\widetilde{J}(\mathbf{k})$ denotes the Fourier transform of the pair coupling Eq. (2b). Setting the coupling constant to $J=0$ the evaluation of this expression yields, the divergent term at $\mathbf{r}=\mathbf{0}$ is excluded,

$$
T_{\mathrm{c}}^{\mathrm{mf}}=\sum_{\mathbf{r} \in \mathbb{Z}^{2}}^{\prime} \frac{\omega_{\|} r_{\|}^{2}+\omega_{\perp} r_{\perp}^{2}}{|\mathbf{r}|^{5}}=\frac{1}{2}\left(\omega_{\|}+\omega_{\perp}\right) \Theta\left(\frac{3}{2}\right)
$$

with the two-dimensional lattice sum [27]

$$
\Theta(s)=4^{1-s} \zeta(s)\left(\zeta\left(s, \frac{1}{4}\right)-\zeta\left(s, \frac{3}{4}\right)\right),
$$

where $\zeta(s, a)$ denotes the generalized Riemann Zeta function. Setting $\omega_{\|}=\omega_{\perp}=1$, the mean-field critical temperature takes the value

$$
T_{\mathrm{c}}^{\mathrm{mf}}=\Theta\left(\frac{3}{2}\right) \simeq 9.0336 .
$$

According to the finite-size scaling relations listed in Sec. III the UFSS functions were evaluated from the MC data and plotted against the temperature scaling variable $x_{\mathrm{rg}}$ (see Fig. 2). The data collapse was achieved by adjusting the critical temperature $T_{\mathrm{c}} \equiv T_{\mathrm{c}}(\infty)$ and the parameters $v$ and $L_{0}$ in the following way. First we determined $L_{0}$ from the requirement that the maximum of the scaled susceptibility $T \chi_{\text {abs }}(t, 0 ; L) L^{-1} \ln ^{-\frac{1}{2}}\left(\frac{L}{L_{0}}\right)$ collapses for different $L$ [Fig. 20] , as this peak height is independent of $T_{\mathrm{c}}$ and $v$. After that we adjusted $T_{\mathrm{c}}$ and $v$ until the scaled cumulant $U(t ; L)$ vs. $x_{\mathrm{rg}}$ [Eqs. (126a)] collapses [Fig. 21] and fits the well known critical value 2, 20]

$$
Y_{U}(0)=1-\frac{\Gamma\left(\frac{1}{4}\right)^{4}}{24 \pi^{2}}=0.27052 \ldots
$$

at $x_{\mathrm{rg}}=0$. Finally, in order to compare the numerical data to the zero mode finite-size scaling functions listed in Sec. III] these functions were fitted to the numerical data by tuning the nonuniversal metric factors $C_{1}$ and $C_{2}$. The values of all parameters as they were determined from this analysis are listed in Table The whole data analysis was done using Fsscale [28].

In addition to the temperature dependence we also studied the dependence of the quantities $m, m_{\mathrm{abs}}, \chi$, and $\chi_{\text {abs }}$ on an external field at the temperature

$$
T_{\mathrm{c}}(L)=T_{\mathrm{c}}(\infty)\left(1+v L^{-1} \ln ^{-\frac{2}{3}}\left(\frac{L}{L_{0}}\right)\right)
$$

that can be regarded as an effective critical temperature of the finite system (see, e.g. , Ref. [19]). Note that $t_{\mathrm{c}}(L)=T_{\mathrm{c}}(L) / T_{\mathrm{c}}(\infty)-1$ corresponds to the value of $t$ for which the shifted reduced temperature $\hat{t}$ as defined in Eq. (6b) and consequently the temperature scaling variable $x_{\mathrm{rg}}$ [Eq. [6a)] vanishes, as $\hat{t}=t-t_{\mathrm{c}}(L)$. Due to this choice the numerical data obtained at this temperature and nonzero fields might then be compared to the corresponding zero mode finite-size scaling functions $Y_{i}^{\mathrm{mf}}(0, y)$ within a finite-size scaling plot. Therefore we stored for each of the system lengths $L=\{16,32,64,128,256\}$ a

\begin{tabular}{|c|c|c|c|c|}
\hline$T_{\mathrm{c}}(\infty)$ & $L_{0}$ & $v$ & $C_{1}$ & $C_{2}$ \\
\hline \hline $8.0302(3)$ & $3.0(2)$ & $1.16(2)$ & $0.735(10)$ & $0.92(1)$ \\
\hline
\end{tabular}

Table I: Values of the fit parameters used throughout this work. 

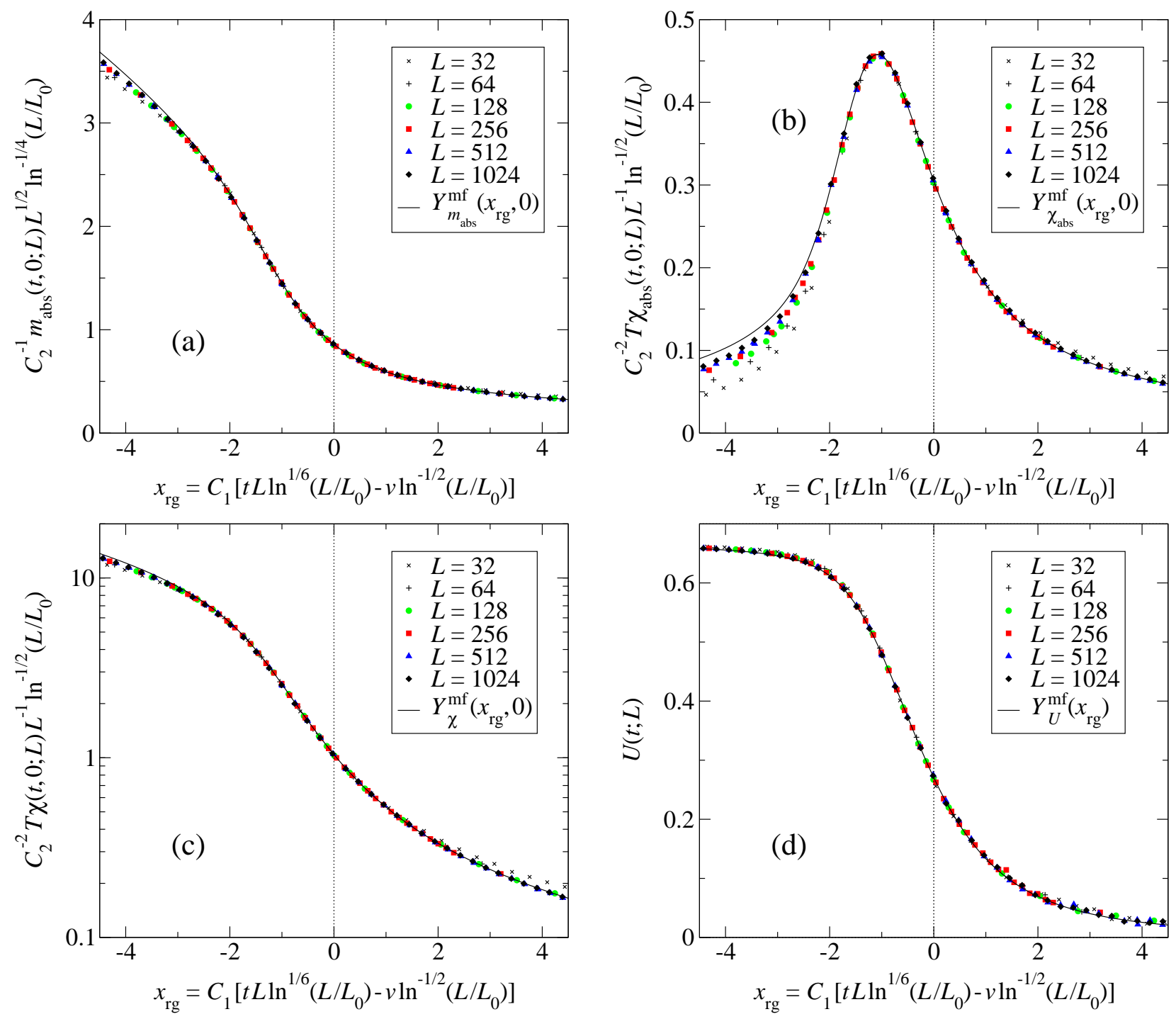

Figure 2: Finite-size scaling plot of the magnetization $m_{\mathrm{abs}}(t, 0 ; L)(\mathrm{a})$, the susceptibility $\chi_{\mathrm{abs}}(t, 0 ; L)(\mathrm{b})$, the susceptibility $\chi(t, 0 ; L)(\mathrm{c})$, and the Binder cumulant $U(t ; L)(\mathrm{d})$, versus scaling argument $x_{\mathrm{rg}}$. The corresponding zero mode finite-size scaling functions $Y_{i}^{\mathrm{mf}}(x, 0)$ are displayed as solid lines. Each data point was obtained by averaging over $10^{5} \mathrm{MCS}$.

magnetization histogram at the corresponding temperature $T_{\mathrm{c}}(L)$ and zero external field, i.e. at $x_{\mathrm{rg}}=y_{\mathrm{rg}}=0$. Using these histograms, the considered quantities were extrapolated to nonzero fields [25] and plotted as implied by their finite-size scaling forms (see Fig. 31). The values of the fit parameters required for these plots are taken consistently as they were determined from the tempera-

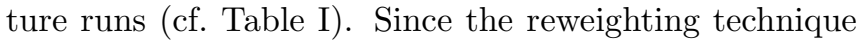
allows the extrapolation of the quantities to arbitrary values of the external field, the data are displayed as continuous lines.

Within the intervals of the scaling variables $x_{\mathrm{rg}}$ and $y_{\mathrm{rg}}$ that were considered in the simulations we find excellent agreement of the MC data with the zero mode theory. In Figs. 2(a,b) merely for negative values of the temperature scaling variable $x_{\mathrm{rg}}$ remarkable deviations occur and the MC data with increasing system size $L$ converge to the corresponding zero mode finite-size scaling function. This effect indicates the presence of further corrections that are expected to vanish in the limit $L \rightarrow \infty$ (see also Ref. 29] that refers to the five-dimensional short-range nearest neighbor Ising model).

The deviations visible in Fig. 3 for large values of the field scaling variable $y_{\mathrm{rg}}$ are due to the finite size of the histograms that were used to obtain the curves shown there by means of the reweighting technique. They originate from the fact that a magnetization histogram of size $K$ that is based on $K$ values of the average spin $\bar{s}$ [Eq. (8)] sampled in a MC simulation, in the mean does not contain values of $\bar{s}$ occurring in a simulation with a lower probability than $1 / K$. As will be demonstrated in the following, these deviations can also be reproduced 

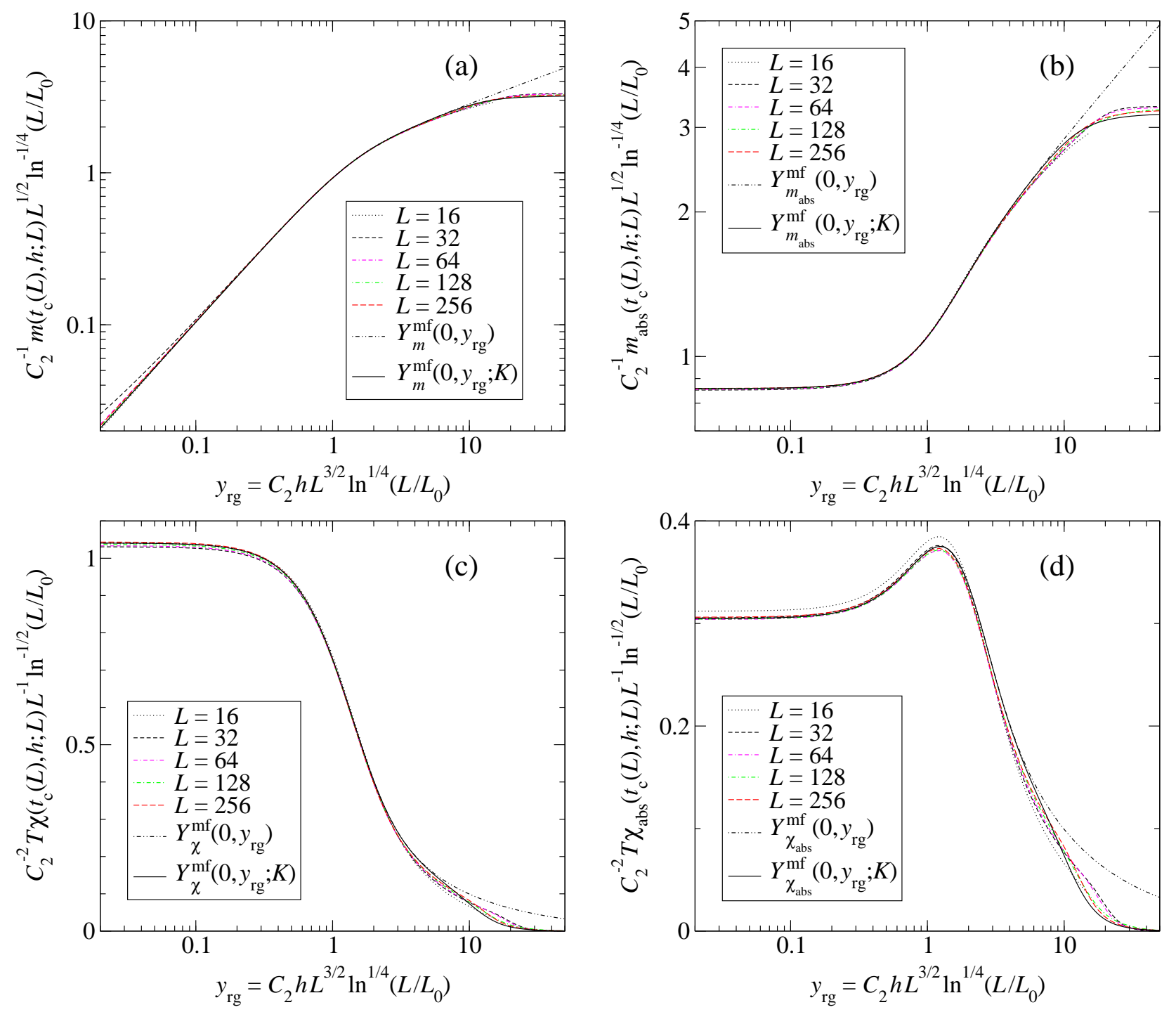

Figure 3: Finite-size scaling plot of the magnetization $m\left(t_{c}(L), h ; L\right)$ (a) and $m_{\mathrm{abs}}\left(t_{c}(L), h ; L\right)$ (b), and the susceptibility $\chi\left(t_{c}(L), h ; L\right)(\mathrm{c})$ and $\chi_{\text {abs }}\left(t_{c}(L), h ; L\right)(\mathrm{d})$, versus scaling argument $y_{\mathrm{rg}}$. The size of the histograms that were used to obtain the curves amounts $K=2 \times 10^{6}$ MCS for each system length $L$. The zero mode finite-size scaling functions $Y_{i}^{\mathrm{mf}}(x, y ; K)$ are plotted as solid lines where the cutoff parameter takes the value $q_{\Lambda}\left(2 \times 10^{6}\right) \simeq 3.2201$ (see text).

within the zero mode theory by truncating the zero mode probability distribution $\mathcal{P}_{0}(\varphi)$, Eq. (16), from which we start and perform the rescaling, Eq. (24). After that we set $x_{\mathrm{mf}}=0$ and $y_{\mathrm{mf}}=0$ since the magnetization histograms we used to obtain the curves shown in Fig. 3 were recorded in the simulations also for vanishing $x_{\mathrm{rg}}$ and $y_{\mathrm{rg}}$. The corresponding normalized zero mode probability distribution denoted as $\mathcal{P}_{0, \mathrm{c}}(\varphi)$ then reads

$$
\mathcal{P}_{0, \mathrm{c}}(\varphi)=\left(u L^{d}\right)^{\frac{1}{4}} Y_{\mathcal{P}_{0, \mathrm{c}}}^{\mathrm{mf}}\left(\left(u L^{d}\right)^{\frac{1}{4}} \varphi\right)
$$

with the zero mode finite-size scaling function

$$
Y_{\mathcal{P}_{0, \mathrm{c}}}^{\mathrm{mf}}(q)=\frac{3^{\frac{3}{4}}}{2 \Gamma\left(\frac{1}{4}\right)} e^{-\frac{27}{256} q^{4}}
$$

and hence decreases monotonically for increasing $|\varphi|$. So to reproduce the effect that is due to the finite size of the histograms we chop the tails of this distribution by limiting the integration range in Eq. (17) and the zero mode partition function, Eq. (15), to a finite interval $\left[-\varphi_{\Lambda}, \varphi_{\Lambda}\right]$. To make an appropriate definition of the cut-off parameter $\varphi_{\Lambda} \equiv \varphi_{\Lambda}(K)>0$ we use an estimation that is known from extreme value statistics [30]. We assume that in the mean only one out of $K$ measured values of the average order parameter $\varphi$ lies outside the interval $]-\varphi_{\Lambda}, \varphi_{\Lambda}[$, i. e. it fulfills $|\varphi| \geq \varphi_{\Lambda}$. This implies

$$
P\left(|\varphi| \geq \varphi_{\Lambda}\right)=2 \int_{\varphi_{\Lambda}}^{\infty} \mathrm{d} \varphi \mathcal{P}_{0, \mathrm{c}}(\varphi) \stackrel{!}{=} \frac{1}{K}
$$

from which the cutoff $\varphi_{\Lambda}$ is implicitly defined. Replac- 


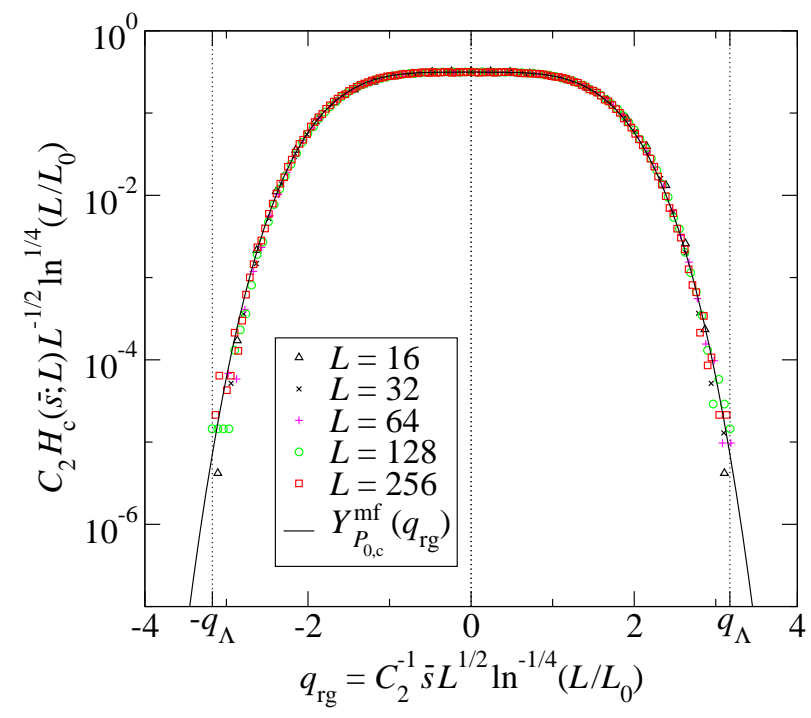

Figure 4: Finite-size scaling plot of the critical histogram $H_{\mathrm{c}}(\bar{s} ; L)$. The size of the histogram depicted here amounts $K=10^{6} \mathrm{MCS}$ for each system length $L$. According to Eq. (42) this corresponds to a cutoff value of $q_{\Lambda}\left(10^{6}\right) \simeq 3.1728$. The value of the fit parameter $L_{0}$ and the nonuniversal metric factor $C_{2}$ are taken as they are listed in Table

ing $\mathcal{P}_{0, \mathrm{c}}(\varphi)$ according to Eqs. (40) the evaluation of the resulting integral yields

$$
2 \int_{q_{\Lambda}}^{\infty} \mathrm{d} q Y_{\mathcal{P}_{0, c}}^{\mathrm{mf}}(q)=\frac{\Gamma\left(\frac{1}{4}, \frac{27}{256} q_{\Lambda}^{4}\right)}{\Gamma\left(\frac{1}{4}\right)} \stackrel{!}{=} \frac{1}{K},
$$

where $q_{\Lambda}=\left(u L^{d}\right)^{\frac{1}{4}} \varphi_{\Lambda}$ and $\Gamma(a, b)$ denotes the incomplete Gamma function. Expanding this expression and separating $q_{\Lambda} \equiv q_{\Lambda}(K)$ yields to leading order a logarithmic dependence on the histogram size $K$ of the form

$$
q_{\Lambda}(K) \stackrel{K \rightarrow \infty}{\sim} \sqrt{\frac{8}{3}}\left(\frac{4}{3} \ln \left(\frac{K}{K_{0}}\right)-\ln \left(\frac{4}{3} \ln \left(\frac{K}{K_{0}}\right)\right)\right)^{\frac{1}{4}}
$$

with the constant $K_{0}=(3 / 4)^{\frac{3}{4}} \Gamma\left(\frac{1}{4}\right)$.

To demonstrate the validity of the definition of the cut-

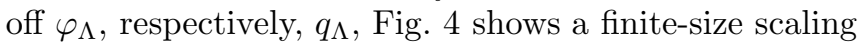
plot of the critical magnetization histogram $H_{\mathrm{c}}$ that was recorded for $x_{\mathrm{rg}}=y_{\mathrm{rg}}=0$. Starting from the finite-size scaling form of the magnetization, Eq. (9a), it is straightforward to show that this histogram scales as

$$
H_{\mathrm{c}}(\bar{s} ; L) \sim C_{2}^{-1} L^{\frac{1}{2}} \ln ^{-\frac{1}{4}}\left(\frac{L}{L_{0}}\right) Y_{H_{\mathrm{c}}}\left(q_{\mathrm{rg}}\right)
$$

with the finite-size scaling function $Y_{H_{\mathrm{c}}}(q)$ and the scaling variable $q_{\mathrm{rg}}=C_{2}^{-1} \bar{s} L^{\frac{1}{2}} \ln ^{-\frac{1}{4}}\left(\frac{L}{L_{0}}\right)$. As is expected also in Fig. 4 the $\mathrm{MC}$ data are found to be in very good agreement with the zero mode expression $Y_{\mathcal{P}_{0, c}}^{\mathrm{mf}}(q)$ [Eq. 40b] of the finite-size scaling function $Y_{H_{\mathrm{c}}}(q)$. It furthermore turns out that all data points lie accurately within the interval $\left[-q_{\Lambda}(K), q_{\Lambda}(K)\right]$ that corresponds to the size of the displayed histogram. The zero mode finite-size scaling functions $Y_{i}^{\mathrm{mf}}(x, y ; K)$ that were evaluated using the truncated zero mode probability distribution with the cutoff $q_{\Lambda}(K)$ are also shown in Fig. 31 and nicely agree with the extrapolated data.

Before finishing this section we also want to discuss the importance of the corrections to the leading orders in the expansions in Eqs. (7) that are determined by the parameters $L_{0}$ and $v$. For that purpose Figs. [5 (a,b) show the finite-size scaling plot of the susceptibility $\chi_{\mathrm{abs}}(t, 0 ; L)$ versus scaling variable $x_{\text {rg }}$ (Fig. [2(b)) where we set $v=0$ in Fig. 5(a) and $L_{0}=1$ in Fig. 5(b). The values of the other parameters entering the plots are taken from Table II As is expected, neglecting one of the corrections causes a significant displacement between the MC data and the zero mode finite-size scaling function $Y_{\chi_{\mathrm{abs}}}^{\mathrm{mf}}\left(x_{\mathrm{rg}}, 0\right)$. This effect could partially be compensated by readjusting the remaining fit parameters, but this procedure would in the case of Fig. [5(a) lead to a wrong determination of the critical temperature $T_{\mathrm{c}}(\infty)$ (see also discussions in Ref. 31 that refer to spin models above the upper critical dimensionality).

\section{CONCLUSIONS}

We have introduced a two-dimensional long-range spin model that displays both isotropic and anisotropic phase transitions and, in particular, strongly anisotropic phase transitions. As a first stage the critical behavior of the model in the isotropic case for which it is found to be at its upper critical dimensionality was investigated. For that purpose we have carried out Monte Carlo simulations and studied the temperature and field dependence of several quantities. Using results of the renormalization group, the numerical data obtained for different system sizes were analyzed by means of a finite-size scaling analysis. It turns out that beside a size-dependent shift that has already been discussed in the literature a characteristic length $L_{0}$ that was inserted to the logarithms is an important correction that must not be neglected.

Furthermore, the collapsed data were compared to the zero mode (mean-field) theory and found to be in excellent agreement. It turns out that the logarithmic corrections typically occurring at the upper critical dimensionality do only enter the finite-size scaling functions through their arguments, whereby these functions were derived from zero mode theory. This shows that at least in the present case the concept of universal finite-size scaling functions can be extended to the upper critical dimensionality.

Finally we note that the numerical results strongly indicate the validity of the zero mode theory at the upper critical dimensionality and might shed new light on recent controversial discussions about its correctness for $d \geq d_{\mathrm{u}}$ 29, 32.

As it will be subject of a future work it is desirable to extend the analysis shown above to the anisotropic 

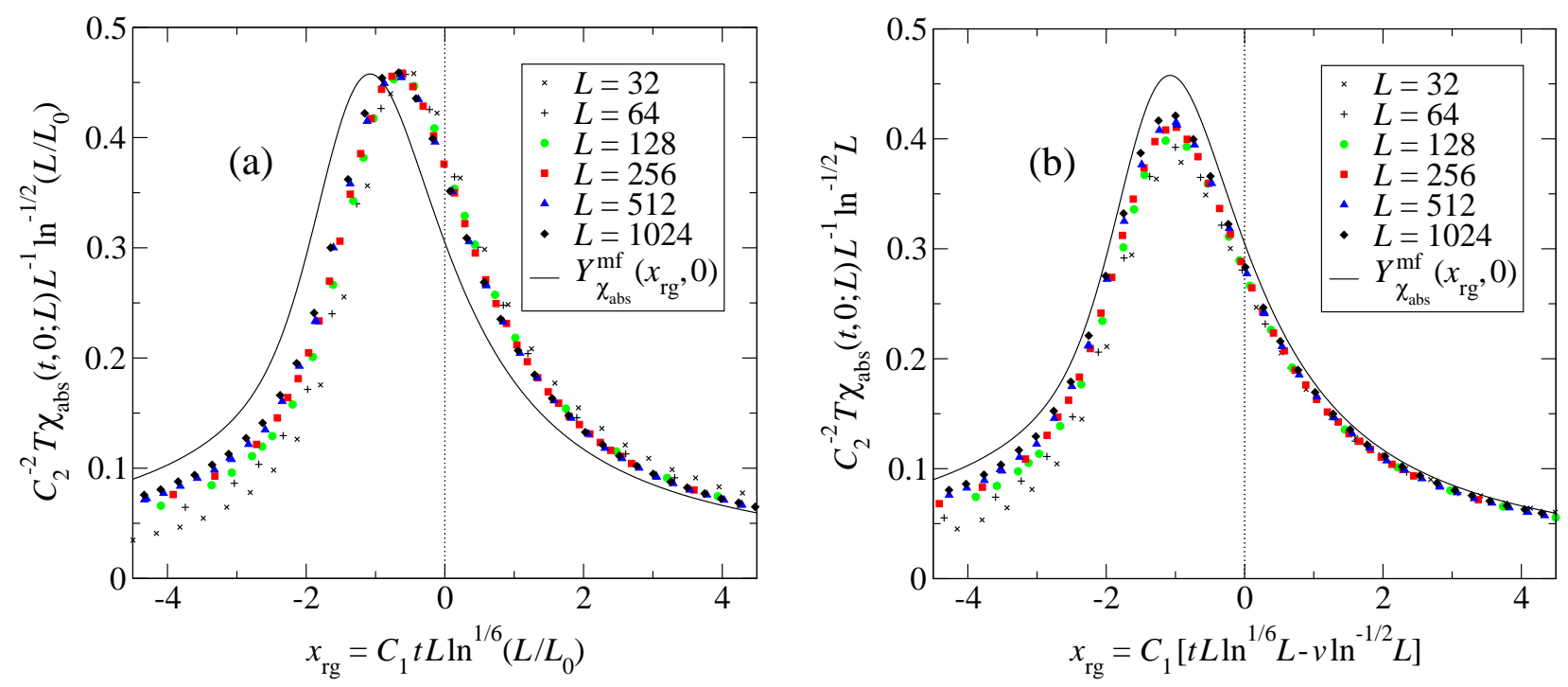

Figure 5: Finite-size scaling plot of the susceptibility $\chi_{\text {abs }}(t, 0 ; L)$ without the shift correction $(v=0)$ (a) and with $L_{0}=1(\mathrm{~b})$.

case $\omega_{\|} \neq \omega_{\perp}$. In particular, the critical behavior of the model should be investigated when approaching the strongly anisotropic cases $\omega_{\|}=-2 \omega_{\perp}$ and $-2 \omega_{\|}=\omega_{\perp}$, respectively.

\section{Acknowledgments}

The authors would like to thank S. Lübeck, Professor K. D. Usadel and Professor H. W. Diehl for valuable discussions.

\section{Appendix A: THE FUNCTIONS $\boldsymbol{\Xi}_{\mathrm{m}}(\mathrm{x}, 0)$ AND $\Xi_{\mathbf{m}}(0, \mathrm{y})$}

The evaluation of the function $\Xi_{m}(\mathrm{x}, 0)$ with MATHEMATICA [33] yields

$$
\begin{aligned}
\Xi_{m}(x, 0)= & 3^{-\frac{3}{4}(m+1)} 4^{m}\left(\Gamma\left(\frac{m+1}{4}\right){ }_{1} F_{1}\left(\frac{m+1}{4} ; \frac{1}{2} ; x^{2}\right)\right. \\
& \left.-2 x \Gamma\left(\frac{m+3}{4}\right){ }_{1} F_{1}\left(\frac{m+3}{4} ; \frac{3}{2} ; x^{2}\right)\right)
\end{aligned}
$$

with the confluent hypergeometric function ${ }_{1} F_{1}(a ; b ; x)$. This expression is valid for $x \in \mathbb{R}$ and $m \in \mathbb{R}^{>-1}$ and can be simplified further for a given integer value of the parameter $m$. For $m=\{0,1,2,4\}$ one obtains

$$
\begin{aligned}
& \Xi_{0}(x, 0)=3^{-\frac{3}{4}} e^{\frac{x^{2}}{2}} \Upsilon_{\frac{1}{4}}(x), \\
& \Xi_{1}(x, 0)=\frac{4}{3} \sqrt{\frac{\pi}{3}} e^{x^{2}} \operatorname{erfc}(x), \\
& \Xi_{2}(x, 0)=\frac{8}{9} 3^{-\frac{1}{4}} e^{\frac{x^{2}}{2}}\left(\Upsilon_{\frac{3}{4}}(x)-x \Upsilon_{\frac{1}{4}}(x)\right), \\
& \Xi_{4}(x, 0)=\frac{64}{81} 3^{\frac{1}{4}} e^{\frac{x^{2}}{2}}\left(2 x^{2} \Upsilon_{\frac{1}{4}}(x)-3 x \Upsilon_{\frac{3}{4}}(x)-\Upsilon_{\frac{5}{4}}(x)\right) \text {, }
\end{aligned}
$$

where the function

$$
\Upsilon_{a}(x)=\pi\left(x^{2}\right)^{a}\left(I_{-a}\left(\frac{1}{2} x^{2}\right)-\operatorname{sgn}(x) I_{a}\left(\frac{1}{2} x^{2}\right)\right)
$$

with the modified Bessel function of the first kind $I_{a}(x)$ has been introduced, and erfc $(x)$ denotes the complementary error function. The function $\Upsilon_{a}(x)$ is well behaved through zero argument for the pertinent noninteger positive values of $a$. Assuming $x>0$, Eq. (A3) simplifies to

$$
\Upsilon_{a}(x) \stackrel{x \geq 0}{=} 2 x^{2 a} K_{a}\left(\frac{1}{2} x^{2}\right) \sin (\pi a)
$$

with the modified Bessel function of the second kind $K_{a}(x)$.

An analogous treatment of the function $\Xi_{m}(0, y)$ results in

$$
\begin{aligned}
\Xi_{m}(0, y)= & \frac{1}{4} \sum_{k=1}^{4}\left(\frac{256}{27}\right)^{\frac{m+k}{4}} \frac{y^{k-1}}{\Gamma(k)} \Gamma\left(\frac{m+k}{4}\right) \\
& \times{ }_{2} F_{4}\left(\frac{m+k}{4}, 1 ; \frac{k}{4}, \frac{k+1}{4}, \frac{k+2}{4}, \frac{k+3}{4} ; \frac{y^{4}}{27}\right)
\end{aligned}
$$

where ${ }_{p} F_{q}\left(a_{1}, \ldots, a_{p} ; b_{1}, \ldots, b_{q} ; x\right)$ denotes the generalized hypergeometric function. This expression is also valid for $y \in \mathbb{R}$ and $m \in \mathbb{R}^{>-1}$ and cannot be simplified further for a given value of $m$ involving less general functions. Finally let us note that the function $\Xi_{m}(x, y)$ fulfills the recursion relations

$$
\begin{aligned}
& \Xi_{m+2}(x, y)=-\frac{8}{3 \sqrt{3}} \frac{\partial}{\partial x} \Xi_{m}(x, y), \\
& \Xi_{m+1}(x, y)=\frac{\partial}{\partial y} \Xi_{m}(x, y)
\end{aligned}
$$

as follows from Eq. (26). 
[1] E. Luijten and H. W. J. Blöte, Int. J. Mod. Phys. C 6, 359 (1995).

[2] E. Luijten and H. W. J. Blöte, Phys. Rev. B 56, 8945 (1997).

[3] E. Luijten, H. W. J. Blöte, and K. Binder, Phys. Rev. E 54, 4626 (1996).

[4] E. Luijten, H. W. J. Blöte, and K. Binder, Phys. Rev. E 56, 6540 (1997).

[5] A. Hucht, J. Phys A: Math. Gen. 35, L481 (2002).

[6] R. M. Hornreich, M. Luban, and S. Shtrikman, Phys. Rev. Lett. 35, 1678 (1975).

[7] W. Selke, in Phase Transitions and Critical Phenomena, edited by C. Domb and J. L. Lebowitz (Academic Press, London, 1992), vol. 15.

[8] H. W. Diehl and M. Shpot, Phys. Rev. B 62, 12338 (2000).

[9] M. Pleimling and M. Henkel, Phys. Rev. Lett. 87, 125702 (2001).

[10] M. B. Taylor and B. L. Györffy, J. Phys: Condens. Matter 5, 4527 (1993).

[11] A. B. MacIsaac, J. P. Whitehead, M. C. Robinson, and K. De'Bell, Phys. Rev. B 51, 16033 (1995).

[12] A. Hucht and D. Grüneberg (2004), in preparation.

[13] M. E. Fisher, S. k. Ma, and B. G. Nickel, Phys. Rev. Lett. 29, 917 (1972).

[14] M. E. Fisher, in Proceedings of the School on Critical Phenomena, Stellenbosch, South Africa, 1982, edited by F. J. W. Hahne (Springer Verlag, Berlin Heidelberg, 1983), vol. 186, chap. 1.

[15] V. Privman and M. E. Fisher, J. Stat. Phys. 33, 385 (1983).

[16] S. Lübeck and P. C. Heger, Phys. Rev. Lett. 90, 230601 (2003).

[17] V. Privman and M. E. Fisher, Phys. Rev. B 30, 322 (1984).
[18] K. Binder, Z. Phys. B 43, 119 (1981).

[19] K. Binder, M. Nauenberg, V. Privman, and A. P. Young, Phys. Rev. B 31, 1498 (1985).

[20] E. Brézin and J. Zinn-Justin, Nucl. Phys. B 257, 687 (1985).

[21] J. Rudnick, H. Guo, and D. Jasnow, J. Stat. Phys. 41, 353 (1985).

[22] A. Aharony, in Phase Transitions and Critical Phenomena, edited by C. Domb and M. S. Green (Academic Press, London, 1976), vol. 6, chap. 6.

[23] M. L. Glasser, V. Privman, and L. S. Schulman, Phys. Rev. B 35, 1841 (1987).

[24] U. Wolff, Phys. Rev. Lett. 62, 361 (1989).

[25] A. M. Ferrenberg and R. H. Swendsen, Phys. Rev. Lett. 61, 2635 (1988).

[26] A. Aharony, in Proceedings of the School on Critical Phenomena, Stellenbosch, South Africa, 1982, edited by F. J. W. Hahne (Springer Verlag, Berlin Heidelberg, 1983), vol. 186, chap. 3.

[27] A. Hucht, A. Moschel, and K. D. Usadel, J. Magn. Magn. Mater. 148, 32 (1995).

[28] F. Hucht, fsscale: A program for doing Finite-Size Scaling (1998-2003), URL http://www.thp.Uni-Duisburg.de/fsscale/

[29] E. Luijten, K. Binder, and H. W. J. Blöte, Eur. Phys. J. B 9, 289 (1999).

[30] S. Kotz and S. Nadarajah, Extreme Value Distributions (Imperial College Press, London, 2000).

[31] E. Luijten and H. W. J. Blöte, Phys. Rev. Lett. 76, 1557 (1996).

[32] X. S. Chen and V. Dohm, Int. J. Mod. Phys. C 9, 1007 (1998).

[33] S. Wolfram, The Mathematica Book (Wolfram Media, Cambridge University Press, 1999), 4th ed. 